\title{
El debido proceso telemático en Venezuela
}

\section{The due telematic process in Venezuela}

\section{O devido processo telemático na Venezuela}

Lisbeth del Carmen Gutiérrez Piña
lgp01_7030@yahoo.es
Primera Instancia de Sustanciación, Mediación y Ejecución del Trabajo de la Circunscripción Judicial Laboral del
Estado Carabobo, Dirección Ejecutiva de la Magistratura, Circuito Judicial Laboral del Estado Carabobo,
Venezuela

Articulo recibido en febrero 2018; arbitrado en marzo 2018; aprobado en abril 2018 y publicado junio de 2018

\section{RESUMEN}

El objetivo del presente fue estudio hacer una análisis práctico de lo que es la aplicación del debido proceso, que forma parte de un proceso escrito, se adecue al uso de las herramientas tecnológicas, donde el solicitante tenga oportuna respuesta teniendo la certeza que su información se encuentra encriptada en un sistema informático que asegure la confidencialidad o la no vulnerabilidad de su información, en la que no sea necesaria o apremiante su presencia en el Juzgado $\mathrm{u}$ oficina que se trate para gestionar su requerimiento, sino que pueda hacerlo desde la comodidad de su casa, oficina, centro de chateo mas cercano, lugar de reclusión, o donde se encuentre, donde se utiliza la metodología descriptiva mediante el análisis de la norma escrita y su ponderabilidad en la practica forense al uso de las herramientas tecnológicas vigentes, en el entendido que el "debido proceso" es una garantía constitucional establecida en el artículo 49 de la Constitución de la República Bolivariana de Venezuela de 1999, reconocida como el derecho humano que tiene toda persona de acceder a los órganos administrativos o judiciales para conocer los hechos por los cuales se le investiga y a defenderse.

Palabras clave: Debido proceso; garantía; herramientas tecnológica, adecuación
The objective of this paper is to develop a practical analysis of the application of due process, which is part of a written process regarding the adequate use of the technological tools, where the applicant receives a timely response with certainty that their information is encrypted in a computer system that assures confidentiality or no vulnerability of said information; of which the applicant's presence in front of a judge or office where the required process is managed is neither necessary nor urgent, but rather that the applicant may complete the process from the comfort of his or her home, office, chat center, place of seclusion, or wherever he or she may be, where a descriptive methodology is utilized through a written analysis and its approval in forensic practices in using current technological tools, as established in the "due process" that is guaranteed by article 49 of the 1999 Constitution of the Bolivarian Republic of Venezuela, recognized as the human right that every person is entitled to access to the administrative or judicial bodies in order to recognize the facts for which they are being investigated and to defend themselves.

Key words: Due process, guarantee, technological tools, adequacy 
RESUMO

O objetivo deste trabalho é fazer uma análise práctica do que é a aplicação do devido processo, que faz parte de um processo escrito sobre o uso adequado das ferramentas tecnológicas, onde o solicitante recebe uma resposta atempada com certeza de que sua informação é criptografada uma num sistema informático que garanta a confidencialidade ou nenhuma vulnerabilidade da referida informação; de qual a presença do requerente repente no Tribunal ou gabinete não é necessária nem urgente para gerir o seu pedido, mas o aplicante pode concluir o processo a partir do conforto da sua casa, escritório, chatroom, local de detenção, onde

\section{INTRODUCCIÓN}

El propósito de esta investigación fue establecer de manera clara el impacto que tuvo el uso de las herramientas tecnológicas en el proceso venezolano, especialmente en el proceso judicial, para la creación y contribución al debido proceso telemático, y con ello la constitución del expediente digital, aun cuando en la actualidad el concepto está siendo revisado, se puede señalar que desde el año 2002, tal adecuación se ido hilvanando de manera tímida pero constante, pues en la actualidad se dificulta acceder a la información un proceso, lámese expediente, sin contar con herramientas básicas como los portales informativos del sistema en red, sistema juris 2000, Interned, y otros.

En la actualidad un grupo de abogados adscritos a la Escuela Nacional de la Magistratura del Tribunal Supremo de Justicia, así como abogados, jueces y demás funcionarios del poder judicial, se han dado a la tarea de evaluar los aspectos positivos y negativos de esta evolución procesal telemática partiendo de los criterios conocidos y experimentados con miras a la creación de un basamento jurídico, donde se establezca de forma expresa los lineamientos a seguir para la creación y constitución del expediente digital. quer que estejam, onde uma metodologia descriptiva é utilizada através da análise escrito e ponderabilidade na práctica forense o uso de ferramientas tecnológicas atuais, no entendimiento de que o "devido processo" é uma garantia constitucional estabelecida no artigo 49 da Constituição da República Bolivariana de Venezuela de 1999, reconhecido como o dereito humano que toda pessoa tenha acesso a órgãos administrativos ou judiciais para conhecer os fatos pelos quais estão investigando e se defender.

Palvras chave: Devido processo, garantia, ferramentas tecnológicas, adequaçâo

Partiendo del postulado constitucional de 1999, artículo 49, donde se estableció que el debido proceso es una garantía constitucional a la par de ser un derecho fundamental, que le permite a todos los justiciables el acceso a un proceso que reúna los requisitos mínimos que lleven a la autoridad encargada de resolverlo a pronunciarse de manera justa, equitativa e imparcial; lo que conlleva una serie de actuaciones necesarias para lograr tal cometido, es por ello, el texto constitucional lo desarrolla como una garantía inherente al ser humano, donde "toda" persona sin distinción de raza, credo, color, podrá acudir a cualquier instancia administrativa $o$ judicial $y$ defenderse en el procedimiento que se lleve en su contra, se le garantice estar informado y notificado de todos los tramites que se lleven a cabo en su contra, conocer las pruebas y gozar de tiempo para preparar y estructurar su defensa, recurrir de la decisión que le sea adversa, contar con la asesoría técnica o asistencia de un abogado, y ello es así, dado que el debido proceso va a apareado del derecho a la defensa y ambos forman parte de la tutela judicial efectiva, y si se lleva de manera digital a través del uso de las herramientas tecnológicas, su expectativa de justicia debe estar apareada a los postulados 
enunciados en el texto constitucional, sin ser afectada su expectativa de justicia.

La Corte Interamericana de Derechos Humanos señaló que el debido proceso "es un medio para asegurar, en la mayor medida posible, la solución justa de una controversia", a lo cual contribuyen "el conjunto de actos de diversas características generalmente reunidos bajo el concepto de debido proceso legal", el cual genera las condiciones que deben cumplirse en función de asegurar la adecuada defensa de aquellos cuyos derechos u obligaciones están bajo consideración judicial".

En lo referente al proceso de contenido "telemático", en Venezuela, es importante mencionar la utilidad del Sistema Juris 2000, el cual se aplicó en los Circuito Judiciales Laborales desde la entrada en vigencia de la Ley Orgánica Procesal Laboral en el año 2003, el cual significó un precedente en cuanto al uso de la tecnología en el proceso, el cual significó adecuar el proceso al uso de las herramientas tecnológicas con el firme propósito de agilizar las actuaciones procesales en el enmarcado constitucional de un estado social de derecho y de justicia, lo cual redundo en un trabajo arduo que significo resolver situaciones día a día, buscando la solución de los conflictos planteados de manera más breve en contraposición al vetusto proceso escrito, formalista, escrito y lento, lo cual redundo en una evolución sistemática del debido proceso que se extendió a todas las esferas y ramas del poder judicial así como la parte administrativa, a partir de la cual se empezó a manejar la idea de un proceso telemático, de manera muy tímida, donde las personas tuvieron acceso a la información de su interés de manera oportuna.

El Sistema Juris 2000, desarrollo de manera armónica un esquema de trabajo donde se agilizaron muchas actuaciones relativas al expediente, empero la tendencia es crear una plataforma capaz de formar el expediente digital, donde toda persona pueda obtener información sobre el estatus de su expediente, sin necesidad de acudir a la sede del Tribunal $u$ oficina, sino que lo pudiese hacer desde la comodidad de su casa, oficina, trabajo o centro de Chateo, y donde tenga la certeza que su información no será vulnerada ni usada en su detrimento.

\section{Importancia del problema}

El debido proceso es una de las garantías constitucionales de mayor relevancia en el país, pues el denota el inicio de una investigación contra una persona que debe defenderse y que debe contar con un procedimiento preexistente, contando con una estructura donde puedan obrar todos los escenarios necesarios tendentes a lograr el desarrollo del proceso, en cuyo caso el interesado debe tener conocimiento de todo cuanto obre en su contra, como lo es, el material probatorio, el ser notificado, el estar asistido de abogado, y todo cuanto sea necesario para entender la trascendencia del porque se le investiga, lo cual definitivamente evolucionó con el uso de los portales informativos creados al respecto por el Tribunal Supremo de Justicia, especialmente con el Sistema Juris 2000, el cual agilizo el acceso a la información del justiciable, y con ello se le acercó al estado social de derecho y de justicia.

En este país la normativa procesal siempre ha sido escrita, contando con una serie de formalismos preestablecidos a partir de los cuales se crea el expediente, administrativo o judicial, el cual con el paso del tiempo ha devenido en un atraso, por el excesivo formalismo que conlleva reposiciones inútiles, y redunda en una pérdida de materiales, utilización de equipos, desgaste humano, lo que motivo a los entes gubernamentales en replantear y adecuar el uso de las herramientas tecnológicas para mitigar tanto formalismo y facilitar así al administrado o justiciable que tenga un mayor y mejor acceso a la justicia, más allá de lo oneroso que representa en la actualidad 
mantener la estructura de una organización gubernamental, es por ello, que se esta desarrollando la creación de una plataforma que contenga un sistema informático en red denominado "Expediente Digital" con todas las formalidades exigidas para garantizar el debido proceso "telemático" y con ello la tutela judicial efectiva.

\section{MÉTODO}

El método utilizado para desarrollar la presente investigación es descriptivo, pues se basó en la apremiante necesidad de adecuar el proceso escrito, excesivamente formalista, al uso de las herramientas tecnológicas con fines de agilizar el acceso a la justicia, partiendo de la jurisprudencia del Tribunal Supremo de Justicia, del precedente del uso del sistema Juris 2000 y de los antecedentes de creación del sistema judicial digital implementado en el derecho comparado.

\section{RESULTADOS}

Desde el año 2003, con la entrada en vigencia de la Ley Orgánica Procesal del Trabajo, un grupo de abogados, jueces y magistrados visionarios le dieron forma a la idea de crear un sistema que agilizara el proceso a través de la información en red, con la creación el Sistema Juris 2000, el cual procesa de manera automatizada la información de un expediente, que sirve de base para llevar un orden cronológico de las actuaciones del mismo y donde el justiciable puede conocer de manera rápida el estatus de su requerimiento sin necesidad de solicitar el físico del expediente y le hace el seguimiento del proceso en sus diferentes etapas hasta su culminación, lo cual significo un antecedente de un expedite digital en Venezuela.

El Tribunal Supremo de Justicia ha venido dictando decisiones relacionadas con el uso de las tecnologías, con la visión de adecuar la normativa procesal vigente, buscando crear las bases para iniciar el proceso virtual, que determine el basamento jurídico tendente a la creación del expediente digital, el cual contaría con las formalidades previstas en las leyes, contenidos en una plataforma, donde se le garantice al justiciable, la validez legal de su proceso, la transparencia, la celeridad procesal, la no vulnerabilidad de su información, la asesoría técnica y jurídica, lo que representaría para el estado, la descentralización de las gestiones, el ahorro de recursos y la simplificación de las actuaciones.

Es así como el uso de la tecnología se ha ido implementando para resolver asuntos en procedimientos especiales, como por ejemplo en un caso de Restitución Familiar, se permitió la comunicación por video llamada vía telefónica para lograr la comunicación entre las partes.

En materia de amparo, se ha permitido el uso del correo electrónico como un aval para presentar demandas, ej. Sentencia № 523 del 9 de abril de 2001, ratificada por la 455 del 2 de julio de 2018, y se han ido dictando sentencias que le dan crédito a la información en red, sin dejar de garantizar a las partes el debido proceso.

\section{DISCUSIÓN}

La Constitución de la República Bolivariana de Venezuela de 1999, consagra el debido proceso como una garantía fundamental, en su artículo 49, estableciendo que tal garantía es aplicable tanto a las actuaciones judiciales como a las administrativas.

En tal sentido estableció, en el numeral 1, lo siguiente:

La defensa y la asistencia jurídica son derechos inviolables en todo estado y grado de la investigación y del proceso. Toda persona tiene derecho a ser notificada de los cargos por los cuales se le investiga, de acceder a las pruebas y de disponer del tiempo y de los medios adecuados para ejercer su defensa. Serán nulas las pruebas obtenidas mediante violación del debido proceso. Toda persona declarada culpable tiene derecho a recurrir del fallo,...

Desde el punto de vista constitucional, el debido proceso fue concebido como la forma a 
través de la cual, el justiciable accede a la justicia, teniendo la certeza que su procedimiento preestablecido esta blindado, teniendo la seguridad que su derecho no podrá ser afectado so pena de nulidad, en el cual gozará de todos los medios necesarios para su defensa, al estar asistido o asesorado de abogado; a obtener información de manera detallada sobre las razones por las cuales se le investiga; conocer las pruebas, disponer del tiempo suficiente para preparar su defensa, recurrir el fallo, en fin gestionar todo cuanto sea necesario para obtener tutela judicial efectiva dentro del debido proceso.

En esta orientación, la Sala Constitucional del Tribunal Supremo de Justicia en Sentencia Nro. 708, del 10/05/2001, estableció la siguiente doctrina que marca un avance en cuanto al punto que se trata:

El derecho a la tutela judicial efectiva, de amplísimo contenido, comprende el derecho a ser oído por los órganos de administración de justicia establecidos por el Estado, es decir, no sólo el derecho de acceso sino también el derecho a que, cumplidos los requisitos establecidos en las leyes adjetivas, los órganos judiciales conozcan el fondo de las pretensiones de los particulares y, mediante una decisión dictada en derecho, determinen el contenido y la extensión del derecho deducido, de allí que la vigente Constitución señale que no se sacrificará la justicia por la omisión de formalidades no esenciales y que el proceso constituye un instrumento fundamental para la realización de la justicia (artículo 257). En un Estado social de derecho y de justicia (artículo 2 de la vigente Constitución), donde se garantiza una justicia expedita, sin dilaciones indebidas $y$ sin formalismos 0 reposiciones inútiles (artículo 26 eiusdem), la interpretación de las instituciones procesales debe ser amplia, tratando que si bien el proceso sea una garantía para que las partes puedan ejercer su derecho de defensa, no por ello se convierta en una traba que impida lograr las garantías que el artículo 26 constitucional instaura.

Así las cosas, con la entrada en vigencia de la Ley Orgánica Procesal del Trabajo, la tendencia es adecuar la normativa procesal vigente, y para ello se ido utilizando el portal informático del Tribunal Supremo de Justicia, el Internet, y el Sistema Juris 2000, siendo este último creado como una herramienta de contenido digital donde el justiciable tiene información sobre el estatus de su expediente, a la par que se implemento la publicación de las decisiones en el portal de la pagina web del máximo Tribunal de la República, donde se demostró que se podía agilizar el proceso, abandonando el excesivamente formalismo escrito, lento y sobre todo oneroso que representaba estar de espalda a la justicia, que en nada garantizaba la tutela judicial efectiva y que vino a sintetizar y coadyuvar el acceso a la justicia de los interesados, lo cual significo un impacto en la estructura judicial imperante en el país para el momento.

El acceso a los expedientes en el sistema judicial venezolano se relacionó con el Sistema Integral de Gestión, Decisión y Documentación JURIS 2000, el cual vino a ser el Modelo Organizacional diseñado e implementado para sistema judicial venezolano y puesto en práctica a partir del año 2002, según Resolución $N^{\circ} 1369$ publicada en la Gaceta Oficial de la República Bolivariana de Venezuela., conforme al cual, la recepción de documentos, demandas, escritos, solicitudes, elaboración de carátulas, asignación del número de los expedientes, las actuaciones judiciales, la publicación de las decisiones, su remisión a otros tribunales, los distintos actos de comunicación, y todas las consultas que tengan que ver con los expedientes, se cumplen, sistema que garantiza seguridad, ya que supone que una vez diarizadas las actuaciones no puede modificarse, con el cual se mejoró el sistema de justicia.

Fue la combinación de la información con la tecnología conectada a un sistema en red la que sirvió de base para llevar a cabo un 
proceso digital que representaba un reto, pues se instruyo a un personal capaz de vaciar la información a un portal, cuyo contenido desarrollaba el orden cronológico de las actuaciones del expediente, desde su presentación por ante la unidad receptora de documentos, hasta su conclusión o ejecución, pasando por las distintas etapas del proceso, lo que incluía los actos de comunicación (notificación), alguacilazgo, fijación de audiencias, fases de Sustanciación, Mediación, Juicio, Superior y Ejecución, con lo cual se simplificaron muchas gestiones y se demostró que un proceso podía durar aproximadamente de 4 a 10 meses, lo cual represento un gran avance, pues existía expedientes con más de 20 años en trámite para el momento.

En la actualidad está latente el tema de adecuar la normativa procesal general a la tecnología vigente, para lo cual se pretende crear el expediente digital en todos los ámbitos procesales sin limitación a la especialidad de la materia que se trate o al ente gubernamental que sea, siempre $y$ cuando se garantice la confiabilidad y no vulnerabilidad de la información en el contenido, con lo cual se tendrían un debido proceso telemático estructurado.

En lo que respecta a la parte administrativa, se observa que las personas se han ido valiendo de algunos de los medios electrónicos o portales creados por el estado venezolano para hacer valer sus pretensiones, ello con el propósito de tener acceso a los procedimientos en los que tengan interés, como por ejemplo, para obtener el Registro de Asegurado, ingresa al portal o página web del Seguro Social, donde aporta sus datos y obtiene la información que requiere de manera oportuna, haciendo uso del derecho de petición (atr.. 51 CRBV), lo que en definitiva representa la garantía constitucional de un proceso debido.

No cabe duda que el avance tecnológico ha llevado a la imperiosa necesidad de que la población deba asígnese de las herramientas existentes para aplicarlas a los procedimientos en curso, respetando para ello la garantía constitucional del debido proceso, el derecho a la defensa y la tutela judicial efectiva, adecuándola tímidamente por vía jurisprudencial, a los casos especiales o aislados, teniendo como resultando sentencias que resuelven los asuntos sometidos a su conociendo, con una motivación propia en cuanto a la valoración y uso de los medios tecnológicos, estando a la espera que se dicten las leyes procesales de contenido digital correspondiente.

En el derecho comparado, por ejemplo el caso de España, que desde el año 2004, implementó el Sistema Lexnet, -recientemente cerrado- en su comienzo fue un sistema de gestión de notificaciones desde los juzgados a los profesionales de justicia (abogados y procuradores), donde las personas afectadas o interesadas tenían acceso a la información del estatus de su expediente a través de una plataforma creada al efecto, pudiendo visualizar su información desde cualquier computadora.

No se puede negar que existen brechas digitales que se deben salvar, empero, en aras de crear o desarrollar un sistema de gestión en red, lo que se pretende es dar inicio a la era moderna del proceso digital en Venezuela, donde se lleve a cabo el debido proceso telemático, en el marco de un expediente digital, con todas las garantías legales necesarias a través de la utilización de las herramientas tecnológicas.

\section{CONCLUSIÓN}

Después de la asistencia de la autora a varias charlas sobre el tema del uso de las tecnologías en el proceso, es importantes indicar que es necesario y urgente adecuar la normativa procesal vigente al uso de las herramientas tecnológicas, dado que la tendencia mundial es el uso del sistema informático en red y ello es importante porque al crearse la plataforma informática, el expediente digital estaría blindado con todas las garantías necesarias para que el justiciable tenga un debido proceso telemático, donde se 
le garantice la confidencialidad, celeridad, inmediatez, concentración, gratuidad, sencillez, brevedad, publicidad, equidad, siendo que el debido y la tutela judicial efectiva, no sean afectados.

A través de esa adecuación el estado deberá crear una base de datos donde el administrado o justiciable pueda acceder $\mathrm{y}$ conocer su procedimiento aportando datos e información vinculante a su persona, partiendo de la confidencialidad, por ejemplo, indicar un domicilio electrónico único que debe aparecer registrado en una base de datos, a partir del cual conoce porque se le investiga, se le pueda notificar, y pueda aportar el material probatorio, conocer el debate probatorio, la decisión, ejercer los recursos, y finalmente saber el estatus de su ejecución.

Es claro que esta visión ésta siendo aplicada por el Tribunal Supremo de Justicia, tímidamente en casos aislados, empero, existe un grupo de juristas que están estructurando el proceso telemático con la visión de simplificar el sistema de justicia, con miras a la solución justa y definitiva de la controversia, en un menor tiempo, con lo cual se estaría ahorrando recursos el estado, sin olvidar que el fin del proceso, es la conservación de la paz social, sin olvidar que el debido proceso, es una garantía constitucional fundamental del Estado de Derecho, dirigida a proteger el respeto de los derechos del justiciable.

\section{Recomendaciones}

Ante la necesidad de cambio y adecuación de las normas procesales, el Debido Proceso establecido como garantía constitucional en concordancia con los artículos 26 y 651, constituye un pilar fundamental para el desarrollo de la tutela judicial efectiva, en el marco de un estado Social, de Derecho y de Justicia, dentro del cual se le garantice a toda persona un proceso eficaz, breve y sobre todo adaptado a la realidad mundial a través del uso de las plataformas informáticas, para lo cual se requiere de manera imperiosa una reforma en todas las materias $y$ especialidades a fin de garantizar un proceso idóneo con personal capacitado donde se estructure el uso de los recursos informáticos existentes sin limitaciones de ninguna índole.

\section{REFERENCIAS}

Constitución de la República Bolivariana de Venezuela, Colección Códigos Básicos, LEGIS, G.O. № 36.860 del 30 de diciembre de 1999, reimpresa en la G.O. № 5.453 Extraordinaria de fecha 24/03/2000. Caracas. Primera Edición, tercera reimpresión.

Corte Interamericana de los Derechos Humanos. Concepto "proceso"

Ley Orgánica Procesal del Trabajo. (2002). Ediciones Paredes, 4ํㅡ Edición, Cánchica B. Jesús M.

Derecho Procesal Español. Sistema Lexnet, (2004) referencia on line.

Sala Constitucional del Tribunal Supremo de Justicia, Sentencia Nro. 708, del $10 / 05 / 2001$

Sala Constitucional del Tribunal Supremo de Justicia, Sentencia № 523 del 9 de abril de 2001, ratificada por la 455 del 2 de julio de 2018. 
Valoración y limitaciones de los medios de pruebas electrónicos en el proceso laboral venezolano

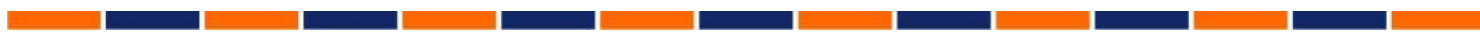

